Aim We set out to establish both the efficacy of the service and levels of patient satisfaction by conducting a survey of our current cohort of 12 IBD patients living in Milton Keynes and receiving treatment with biologics at our unit.

Subjects and Methods Our survey included the evaluation of data from electronic patient records (EPR) and telephone interviews. All patients have experience of the local service although three are presently on a regimen only available at the tertiary centre.

We looked at several aspects of their care including current choice and length of biologic treatment, evidence of remission, occurrence of adverse reactions, monitoring parameters and outpatient follow up arrangements.

Our telephonic patient questionnaire focused on whether families felt they were given sufficient information about treatment, the impact of receiving treatment locally and overall satisfaction with the service.

Results Of our 12 patients with IBD, 2 were diagnosed with Ulcerative Colitis and 10 with Crohn's disease. All are children of school age with an age range from 7 to 17.5 years.

9 children are eligible for local treatment, 7 of them receive Adalimumab injections and 2 children are treated with Infliximab infusions. The 3 children treated with Vedolizumab or Ustekinumab have previously received at least one other biologic locally. Treatment duration ranges from 2- 49 months on their current biologic, with a mean duration of 16.5 months.

6 children started their treatment at MKUH, 3 at Oxford but continued at MKUH. The remaining 3 started at Oxford to continue locally but went back to Oxford due to treatment failure. Initial biologic therapy had to be switched in 6 children due to suboptimal response. All families reported their child as now clinically well. Only 5 children achieved Calprotectin levels below $200 \mu \mathrm{g} / \mathrm{g}$. The mean CRP was 1.4 . Monitoring is done regularly with quarterly reviews at the joint IBD clinic.

All families feel they were given adequate information about treatment and accessible contact points for queries. Formal training was given to all families administering Adalimu$\mathrm{mab}$ at home by local community nursing team. Easy access to all health care professionals involved in the service was especially praised and appreciated. Parents feel the service is patient centred and individualised. There were no serious adverse events. Families described that local access meant less disruption to their child's lifestyle and education.

Summary and conclusion Treatment with biologics at secondary care hospital is safe, effective and contributes significantly to patient satisfaction.

Families feel well supported and appreciate the personalised care and accessibility of the service.

\section{P44 QUALITY IMPROVEMENT: IMPACT OF IMPLEMENTATION OF REFERRAL PATHWAY FOR ABDOMINAL PAIN ON INITIAL ASSESSMENT TIME, BLOOD INVESTIGATIONS REQUESTED, IMAGING PERFORMED AND EVENTUAL OUTCOME}

${ }^{1}$ Hina Rizvi, ${ }^{2}$ Chui Lai, ${ }^{1}$ Vijay Iyer, ${ }^{1}$ Anjum Gandhi. ${ }^{1}$ Birmingham Heartlands Hospital; ${ }^{2}$ Birmingham Women's Hospital

10.1136/flgastro-2021-bspghan.53
Background In our trust, historically paediatric patients presenting with abdominal pain raising suspicion of appendicitis were referred to surgeons and assessed in the Paediatric Assessment Unit. These patients faced long wait times for management plan by surgeons:

- covering theatres/adult wards and intensive care

- waiting for blood and imaging investigation results before finalizing a plan.

This led to delays and affected flow of patients through the PAU and the ward capacity. The Paediatric team decided to bring about a change in the abdominal pain referral pathway by proposing to see all patients referred with abdominal pain and refer to surgeons if deemed appropriate.

We collected data over 2 periods: 2018 and 2020, with the implementation of the new pathway in 2019

Aim To assess:

- Time to first review by a team (paediatric vs surgical)

- Percentage of patients

- With abdominal pain referred to each team

- Who had blood tests and imaging requested by each team

- Discharged, observed, referred and admitted by each team

Methods

- Sample period: 4 weeks in 2018 and 6 weeks in 2020

- Inclusion criteria: all paediatric patients referred with abdominal pain to PAU

- Exclusion criteria: anyone with previous appendectomy or re attending.

- Data collection: retrospective.

- Case notes: reviewed for referral record, review times, and initial diagnosis

- Electronic patient management system: reviewed for blood test and imaging investigation requested and discharge summaries

Results Primary care referral rose from $36 \%$ in 2018 to $47 \%$ in 2020 with a concurrent shift in ED referral from 53\% to $37 \%$ in 2020 showing the effect of the new pathway. $26 \%$ of referrals were made to Paediatric team in 2018 vs $87 \%$ in 2020

Majority of patients were seen within 4 hours by the Paediatric team in both episodes $86 \%$ vs $85 \%$. There was very slight improvement in Surgical team review time $78 \%$ vs $80 \%$.

There was an increase in discharges to $58 \%$ by the surgical team in 2020 from $35 \%$ in 2018. However, we also saw the increase in referral by the Paediatricians to Surgeons from $7 \%$ to $33 \%$

There was an impressive drop in blood investigation requested by the Surgical team from 90\% to 58\%. Requests for imaging by surgical teams declined from $33 \%$ to $17 \%$.

$88 \%$ of the referred patients with abdominal pain had medical diagnosis at discharge and did not need any surgical intervention

Summary Re-auditing after implementing new abdominal pathway shows:

- Overall patient flow through PAU has improved as more patients are being discharged.

- Appropriate reduction in laboratory investigation 2018 vs 2020 (both by surgical team from $90 \%$ to $58 \%$ and Paediatric team from $43 \%$ to $17 \%$ ) 
- Optimization of imaging resources by surgical team from $33 \%$ to $17 \%$

- $88 \%$ of patients with abdominal pain had a medical diagnosis at discharge.

Conclusion

- Abdominal pain is a common presentation in the paediatric population, mostly benign and self-limiting.

- Abdominal pain should be assessed by general paediatricians first and then referred to surgical colleagues if deemed appropriate to avoid unnecessary investigations and imaging.

\section{P45 REVIEW OF DIAGNOSIS AND MANAGEMENT OF COELIAC DISEASE IN A DISTRICT GENERAL HOSPITAL IN THE NORTH WEST}

Ban Alkaaby, Zahra Khan, Suparna Dasgupta. Macclesfield General Hospital

10.1136/flgastro-2021-bspghan.54

Background The guidance for Coeliac Disease (CD) has evolved over the last few years. The highlight of the ESPGHAN guidelines from January 2020 is that high serological markers (tTG and EMA) are now the requirements for confirmation of $\mathrm{CD}$ regardless of symptoms.

Aims

1. To review management of patients and compare it with the latest ESPGHAN/BSPGHAN guidelines.

2. To review time to confirmed diagnosis from the start of symptoms.

3. To look at provision of Gluten free products at schools and Primary Care service.

Methods Retrospective data collected from Coeliac database from January 2018 till November 2020 in a District General Hospital with provision for a dedicated Coeliac Clinic supported by a Specialist Dietician. We reviewed the presentation of symptoms, methods of confirmation of CD, time duration from onset of symptoms till diagnosis, provision of gluten free meals at schools and availability of gluten free products on prescription.

Results 35 patients were diagnosed with CD, 31 were symptomatic and 4 were asymptomatic.

$27 / 31$ of the symptomatic patients had high level tTG (>10 times the upper limit), 20 had positive EMA and HLA DQ2/DQ8 for positive confirmation. The two negative EMA were referred for biopsy.

Three had HLA typing but had high tTG on 2 separate occasions which confirms diagnosis. 2 had only one high tTG and HLA but no EMA or second high tTG so they did not meet the diagnostic criteria.

4 symptomatic patients with low tTG $<10 \mathrm{x}$, had biopsy confirmation. 4 asymptomatic patients had high tTG and +ve EMA.

Of 28 patients referred through Primary Care, 7 were screened and referred with symptom duration of circa 4 to 12 months, another 7 were diagnosed through screening due to positive family history or type 1 Diabetes Mellitus and 14 cases had no specific duration of symptoms recorded. Remaining seven were diagnosed by hospital paediatricians due to different presentations.

Most parents stated that they were providing packed lunch box even if the school provided some gluten free meals as the menu choice lacked variety. Patients with Type 1 diabetes found a packed lunch easier for carbohydrate counting. Parents felt tailor made menu recommendation from the dietician to the school would be beneficial for families and the school.

Conclusion and Recommendation We thus identified that $82 \%$ $(n=29)$ of patient had met the criteria for diagnosis of CD as per 2015 guidelines, but if 2020 guidelines were applied then $94.2 \%(n=31)$ would have meet the criteria.

Recommendations

1. Identifying duration of time needed for the child to be screened will help to raise awareness within primary care practice. This will be audited in the future.

2. There is a large knowledge gap in schools about CD and the importance of convenient access to gluten free meals in enhancing compliance with gluten free food in children. Offering tailor made presentations to the local schools will address this issue. A further review to identify if similar knowledge gap exists in schools regionally is planned.

\section{P46 REVIEW THE DIAGNOSIS OF IBD IN CHILDREN WITH AILD (AUTO IMMUNE LIVER DISEASE) -8 YEARS' EXPERIENCE IN A TERTIARY CENTRE}

Maria Misiou, Ben Hope, Huey Miin Lee, Marianne Samyn, Babu Vadamalayan. King's College Hospital, London

\subsection{6/flgastro-2021-bspghan.55}

Background The association of autoimmune liver disease (AILD) and inflammatory bowel disease (IBD) is well documented. IBD affects about $45 \%$ of children with autoimmune sclerosing cholangitis (AISC) and about 20\% of those with autoimmune hepatitis (AIH). The aim of this study was to describe the clinical features of AILD associated with IBD and to evaluate the role of fecal calprotectin and the time to look for IBD in patients with primary diagnosis of AILD.

Methods We have conducted a retrospective review of paediatric patients with primary diagnosis of AILD and IBD between 2010 and 2018. Patients who were referred or diagnosed with IBD first were excluded. Diagnosis of IBD was based on clinical history, endoscopic appearance and histology findings. AILD patients were classified to AIH or AISC according to histology, radiology results and circulating antibodies. Patients' demographics, symptoms, FC, blood tests, timing before IBD diagnosis and treatment were collected.

Results 114 patients with AILD were identified: 74 (64\%) had screening with FC, 48/74 (64\%) had abnormal and 26 (35\%) normal test. $67 \%$ of FC performed at the diagnosis of liver disease. 48 patients had at least one endoscopy, 25 $(52 \%)$ were diagnosed with IBD, 8 patients had normal endoscopy but positive FC of mean value 162, (range 60332). 15 had no endoscopy despite abnormal FC. 6/26 patients with normal FC underwent endoscopy which was normal. Reason was persistently elevated liver enzymes, relapse of AILD or ongoing bowel symptoms. 25/114 (21\%) patients were diagnosed with IBD, 14/25 (56\%) had AISC, the rest $\mathrm{AIH}$. 25\% had family history of autoimmunity. Mean FC at diagnosis of IBD was 646 (range 60-4004). 14 were males and the mean age at diagnosis was 10 years. 18/ 25 (78\%) had ulcerative colitis (UC), 2/25 (0.8\%) Crohn's disease and $5 / 25(2 \%)$ indeterminate colitis(IBDU). 12/14 